\title{
The Tissue Inhibitor of Metalloproteinases-3 Gene in Breast Carcinoma: Identification of Multiple Polyadenylation Sites and a Stromal Pattern of Expression
}

\author{
Jennifer A. Byrne,* Catherine Tomasetto, ${ }^{*}$ Nicolas Rouyer, ${ }^{\dagger}$ \\ Jean-Pierre Bellocq, ${ }^{+}$Marie-Christine Rio,* and Paul Basset* \\ *Institut de Génétique et de Biologie Moléculaire et Cellulaire, \\ CNRS/INSERM/ULP, Illkirch Cedex, C. U. de Strasbourg, France \\ †Service d'Anatomie Pathologique Générale, Centre Hospitalier \\ Universitaire, Hôpital de Hautepierre, Strasbourg Cedex, France
}

\begin{abstract}
Background: Tissue inhibitor of metalloproteinases-3 (TIMP3) is the third member of the TIMP family of proteins, believed to play a significant role in controlling extracellular matrix remodeling.

Materials and Methods: Differential screening of a human breast carcinoma cDNA library using substracted and PCR-amplified CDNA probes identified a 4.6-kb TIMP3 cDNA, which was used for further cDNA library screenings, Northern blot hybridizations, and the synthesis of riboprobes for in situ RNA hybridization analyses.

Results: The 4.6-kb full-length TIMP3 cDNA contains $3.7 \mathrm{~kb}$ of $3^{\prime}$-untranslated sequence. Additional TIMP3 cDNAs subsequently identified were colinear with the original sequence, but revealed use of four different polyadenylation signals within the $3^{\prime}$-untranslated re-
\end{abstract}

gion, which accounted for the 4.6-, 2.7-, 2.5-, and 2.1-kb TIMP 3 transcripts noted in this and in previous studies. In situ RNA hybridizations demonstrated that in breast carcinoma the TIMP3 gene was predominantly expressed by fibroblastic cells within the tumor stroma adjacent to cancer cells. TIMP3 transcripts were also strongly detected in fibroblastic decidual cells of pregnant endometrium.

Conclusions: Modulating the length of the 3 -untranslated region may represent a mechanism by which TIMP3 gene expression is controlled in tissues. The strong expression of the TIMP3 gene by fibroblastic cells in breast carcinoma supports the importance of tumor stroma as a source of factors influencing human carcinoma growth and progression.

\section{INTRODUCTION}

TIMP3 represents a novel member of the tissue inhibitor of metalloproteinases (TIMP) protein family, previously comprised of TIMP1 and TIMP2 proteins (1). The chicken TIMP3 sequence, which was the first to be identified, shows 28 and $42 \%$ amino acid identity with consensus TIMP1 and TIMP2 amino acid sequences, respectively (1), and is approximately

Address correspondence and reprint requests to: Paul Basset, IGBMC, BP 163, 67404 Illkirch Cedex, C. U. de Strasbourg, France. as similar to TIMP2 as TIMP1 and TIMP2 are to each other (2). All TIMP proteins so far identified function as inhibitors of the matrix metalloproteinase family of enzymes. The physiological role of these enzymes is believed to be the degradation of extracellular matrix components, with matrix metalloproteinase activity being implicated in both physiological and pathological tissue remodeling processes (3-5). TIMP function appears vital for correct regulation of matrix metalloproteinase activity (6), and in the case of TIMP3, elevated gene expression (7) and coding sequence mutations (8) have been recently re- 
ported in two retinal disorders characterized by increased local remodeling of the extracellular matrix.

Either as a consequence or independent of their functions as inhibitors of matrix metalloproteinases, TIMPs affect cellular growth (9). TIMP1 was initially known as erythroid-potentiating activity due to its ability to stimulate colony formation by erythroid precursors (10), and has been shown to stimulate the growth of a variety of cells in culture (11). Erythroid-potentiating activity has been subsequently demonstrated for TIMP2 (12), which has also been found to inhibit basic fibroblast growth factor-induced endothelial cell proliferation (13). TIMP3 is especially interesting in this regard, having been found to possess tumor-promoting properties, such as the ability to promote the detachment of transformed chicken embryo fibroblasts from the extracellular matrix (14).

TIMP3 cDNAs have been recently isolated from chicken (1), mouse (15-17) and human $(7,18-22)$. However, the lengths of the human cDNA sequences published to date do not correspond with the sizes of the 3 most prominent human TIMP3 transcripts noted in previous studies $(18-20,22)$, although a sequence approximating the length of the largest transcript has been reported in the mouse (16). Furthermore, although TIMP3 transcripts are detected in samples of oligodT-selected RNA (17-19), several of the TIMP3 cDNAs described do not have polyA sequences $(1,7,15,17,18)$, suggesting that these are incomplete at least at their $3^{\prime}$ ends. It has been suggested that multiple TIMP3 transcripts arise by alternative splicing involving coding and/or noncoding regions, further processing of the longest transcript, the use of alternate polyadenylation sites, or that the presence of multiple TIMP3 transcripts may actually represent the existence of additional, highly homologous genes $(18-20,22)$. In the absence of cDNA clones corresponding to the most frequently observed TIMP3 transcripts, it is currently unclear which of these mechanisms operate in vivo.

In the course of a differential screening procedure designed to isolate genes that are commonly overexpressed in human carcinomas, we identified a number of cDNAs that were differentially expressed between breast carcinomas and fibroadenomas (23). Following DNA sequencing, one cDNA clone containing a $4.6-\mathrm{kb}$ insert was found to encode human TIMP3. Unlike human TIMP3 cDNAs previously reported, its length corresponded to that of the longest and most predominant TIMP3 transcript in human and mouse RNA samples (7,15-20). A second screening of the breast carcinoma cDNA library and an additional library constructed using metastatic lymph node tissue from a breast cancer patient, identified other TIMP3 clones. The analysis of 5 cDNA clones revealed that 4 distinct polyadenylation sites are used.

In situ RNA hybridization analyses were performed on paraffin-embedded tissue sections to determine the cellular localization of TIMP3 gene expression in human tissues shown to express the gene by Northern blot analysis. TIMP3 transcripts showed strong expression in fibroblastic cells of tumor stroma in all of 14 breast carcinomas examined, whereas normal breast glandular epithelium and cancer cells were weakly and less frequently labeled. This pattern of expression in breast carcinomas further supports the concept that, by synthesizing factors capable of modulating extracellular matrix characteristics, tumor stroma is an important determinant of tumor growth and progression.

\section{MATERIALS AND METHODS Isolation of TIMP3 cDNAs}

The differential screening procedure that led to the cloning of a $4.6-\mathrm{kb}$ TIMP3 cDNA was as follows. Briefly, single-stranded breast carcinoma cDNA was subtracted twice using polyA+ RNA from fibroadenoma (a benign breast lesion) and normal skin. The remaining single-stranded cDNA was then positively selected using skin basal cell carcinoma polyA + RNA, in order to enrich for sequences expressed in both breast and another carcinoma type. Single-stranded cDNA and cDNA-RNA heteroduplexes were separated following the hybridization steps (performed in $4 \mu \mathrm{l} 120 \mathrm{mM}$ sodium phosphate buffer $\mathrm{pH} 6.8$, for $24 \mathrm{hr}$ at $68^{\circ} \mathrm{C}$ ) on hydroxyapatite columns at $60^{\circ} \mathrm{C}(24)$. The cDNA-polyA+ RNA heteroduplex resulting from the first subtraction step was used as a negative probe, since this represented sequences expressed in both malignant and benign tissues, while the heteroduplex resulting from the final positive selection step between subtracted breast carcinoma cDNA and basal cell carcinoma polyA+ RNA was used as a positive, carcinoma-specific probe.

Using both random-primed and oligo-dT primed cDNA from the same breast carcinoma used as a source of the subtracted probes, a breast 
carcinoma cDNA library was constructed in the $\lambda$ ZAP II vector (Stratagene, La Jolla, CA). Sixty thousand plaque forming units (PFUs) from this cDNA library were differentially screened, and a total of 16 genes overexpressed in breast carcinoma as compared with fibroadenoma were identified (23). One of these (S6) was found to correspond to a $4.6-\mathrm{kb}$ TIMP3 cDNA. The S6 cDNA was used to rescreen 150,000 PFUs from the same library, and 500,000 PFUs from a second cDNA library constructed in the $\lambda$ ZAP II vector using oligodT-primed cDNA from a metastatic lymph node of a breast cancer patient. The in vivo excision system for rescuing $\lambda$ ZAP II inserts as pBluescriptSK ${ }^{-}$plasmids was followed according to the manufacturer's instructions (Stratagene, La Jolla, CA).

\section{DNA Sequencing}

Minipreparations of plasmid DNA, further purified by $\mathrm{NaCl}$ and polyethyleneglycol 6000 precipitation, were sequenced with Taq polymerase and either $\mathrm{T} 3$ and/or $\mathrm{T} 7$ primers, or internal primers, and dye-labeled ddNTPs for detection on an Applied Biosystems 373A automated sequencer. Sequence analyses were performed using the GCG sequence analysis package, with sequence homologies being identified using the fastA program by searching the complete combined GenBank/EMBL databanks and, in the case of translated sequences, by searching the complete Swissprot database. The S6 CDNA was completely sequenced in both directions, and other TIMP3 clones subsequently isolated were completely sequenced in at least one direction.

\section{RNA Analyses}

The human cell lines used in this study are described in the American Type Culture Collection catalog (7th Ed., 1992), and surgical specimens were obtained from the Hôpitaux Universitaires de Strasbourg. Cells were cultured until confluent in Dulbecco's modified Eagle's medium supplemented with $10 \%$ fetal calf serum, and surgical specimens stored in liquid nitrogen, before total RNA was isolated as previously described (25). Northern blot analyses were performed with $8 \mu \mathrm{g}$ of total RNA or $500 \mathrm{ng}$ of polyA+ RNA (selected using oligodT cellulose spun-column chromatography provided by the Pharmacia mRNA Purification Kit) electrophoresed through $1.2 \%$ denaturing agarose gels and transferred to nylon membranes (HybondN, Amersham Corp.,
Arlington Heights, IL, U.S.A.). Northern blot hybridizations were performed using the ${ }^{32} \mathrm{P}$-labeled S6 TIMP3 cDNA (see above), and subsequently using that of 36B4, a gene known to be ubiquitously expressed (26). These and following washing steps were performed essentially as described (25), and filters were exposed to Kodak XAR film for $6 \mathrm{hr}$ to 3 days at $-80^{\circ} \mathrm{C}$.

In situ hybridizations were performed on formaldehyde-fixed paraffin-embedded human tissue sections as described (25). Briefly, 5- $\mu \mathrm{m}$ sections that had been deparaffined and acidtreated were treated with proteinase $\mathrm{K}$ and hybridized overnight at $55^{\circ} \mathrm{C}$ with sense or antisense TIMP3 transcripts labeled with ${ }^{35} \mathrm{~S}$-CTP. Transcripts were synthesized using either $\mathrm{T} 3$ or T7 polymerases and plasmid DNA templates purified from two $\mathrm{CsCl}$ gradients and digested with appropriate restriction enzymes. Two cDNAs were used to synthesize probes: the 4579 bp S6 cDNA including 3666 bp of 3'-UTR sequence; and a 1976 bp S6 TIMP3 subclone including the S6 $5^{\prime}$-untranslated region (UTR) and coding sequences, but only $1063 \mathrm{bp}$ of 3'-UTR sequence (see Results). Hybridizations were followed by RNAse treatment as described (25), and washing ( $2 \times \mathrm{SSC}, 50 \%$ formamide, at $55^{\circ} \mathrm{C}$ for $2 \mathrm{hr}$ ), before autoradiography using NTB2 emulsion (Kodak, Rochester, NY, U.S.A.) at $4^{\circ} \mathrm{C}$ for 14 to 32 days. After development, sections were counterstained with toluidine blue.

\section{RESULTS}

\section{Isolation of the S6 Clone and of Additional Homologous Clones}

The cDNA clone containing an insert of $4.6 \mathrm{~kb}$, originally identified in the differential screening procedure, was given the laboratory designation of S6. Use of this clone in Northern blot hybridizations resulted in the frequent detection of 3 transcripts of 4.6, 2.7, and $2.5 \mathrm{~kb}$ in length (Fig. 1). These were strongly expressed in the breast carcinoma from which both the cDNA library and the probes used for differential screening were derived, but were only weakly expressed in the breast fibroadenoma used to subtract the breast carcinoma cDNA. The 3 transcripts were consistently detected together in most human tissues and cell lines examined, although the largest transcript was missing in $\mathrm{HeLa}$ and Caco-2 cells (Fig. 1). In addition to the 2.7- and $2.5-\mathrm{kb}$ transcripts, $2.1-$ and $1.8-\mathrm{kb}$ transcripts 


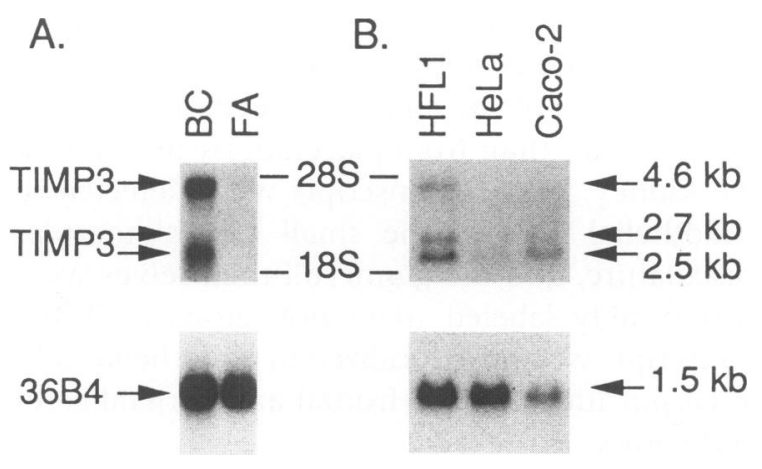

FIG. 1. Northern blot analyses of TIMP3 mRNAs using (A) 500 ng polyA + RNA or (B) 8 $\mu g$ total RNA from human tissues and human cell lines

Panel A indicates that TIMP3 mRNAs were present at higher levels in the breast carcinoma (BC) tissue used both in cDNA library and differential probe construction than in the fibroadenoma (FA) used to subtract breast carcinoma cDNAs. Panel B indicates that while 4.6-, 2.7-, and 2.5-kb TIMP3 transcripts (corresponding to cDNA clones S6/S10, S5, and S4, respectively) were detected in HFLl fibroblasts, the 4.6-kb TIMP3 transcript was not detected in HeLa and Caco- 2 cell lines. Blots were rehybridized with 36B4 cDNA (25), corresponding to a gene ubiquitously expressed. Autoradiographic exposures were for 19 and $6 \mathrm{hr}$ for TIMP3 and 36B4, respectively (A), and for 3 days for both TIMP3 and 36B4 (B).

were also detected in the metastatic lymph node from which the second cDNA library screened in this study was obtained (data not shown).

The length of the $4.6-\mathrm{kb}$ insert of the S6 clone suggested that this represents a full-length cDNA clone corresponding to the largest transcript detected by Northern blot analysis (Fig. 1). The breast carcinoma cDNA library and the cDNA library derived from a metastatic lymph node of a breast cancer patient were screened to isolate additional independent cDNA clones, particularly those corresponding to the smaller transcripts. The results indicated that clones with homology to S6 were present at frequencies of $6 / 10,000$ clones in the breast carcinoma cDNA library, and 8/100,000 clones in the metastatic lymph node cDNA library. On the basis of restriction maps of 10 of the clones isolated, 4 clones were selected for sequence analysis. One clone $(\mathrm{S} 10,4608 \mathrm{bp})$ isolated from the breast carcinoma library, was very similar to S6 $(4579 \mathrm{bp})$. S10 was found to possess an additional $30 \mathrm{bp}$ at its 3' extremity, $22 \mathrm{bp}$ of which represented a polyA sequence, and an additional $10 \mathrm{bp}$ at its $5^{\prime}$ end. The 56 sequence, plus these additional se-

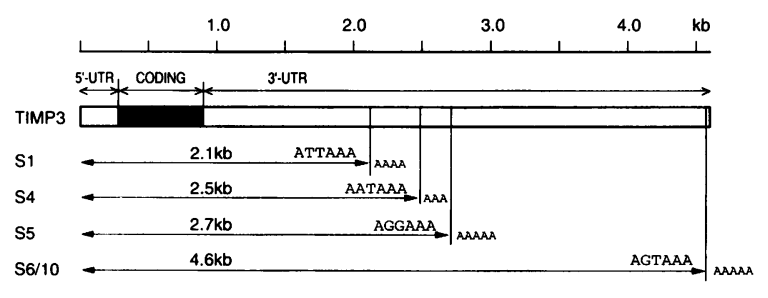

FIG. 2. Structures of the human TIMP3 transcripts

The 5'-UTR, coding sequence, and 3'-UTR of the 4.6-kb human TIMP3 transcript are illustrated diagrammatically at the top of the figure; the vertical lines through the $3^{\prime}$-UTR indicate the positions of functional polyadenylation signals identified by cloning the S1, S4, S5, S6, and S10 (S6/10) cDNAs. The sequence of each polyadenylation signal is indicated above a horizontal line representing the predicted length of the TIMP3 transcript relative to the $4.6-\mathrm{kb}$ TIMP3 sequence. Note that the 3'-UTR occupies 56$80 \%$ of the four TIMP 3 transcripts shown. The TIMP3 2.1-kb transcript in the present study was previously reported as a $1.8 \mathrm{~kb}$ transcript $(21)$. The $\mathrm{S} 6 / 10$ sequence, annotated so as to indicate the positions of the 4 polyadenylation signals identified, has been deposited in the GenBank database under the accession number U14394.

quences from $S 10$, will be referred to hereafter as the S6/10 sequence (Fig. 2). Sequences of the remaining 3 clones, S1 (811 bp), S4 (1917 bp), and S5 (1422 bp), isolated from the metastatic lymph node cDNA library, were contained within that of $S 6 / 10$. All sequences were almost entirely identical within their regions of overlap, with minor variations being noted in $3^{\prime}$-UTR sequences only.

\section{The Structure of the Human S6/10 TIMP3 CDNA}

The identity of $56 / 10$ as a TIMP3 cDNA was indicated by the almost complete homology to shorter human TIMP3 cDNAs previously reported $(7,18-22)$. Despite minor differences in the $5^{\prime}$-UTR $(19,22), 3^{\prime}$-UTR $(7,21)$, and coding $(18,19,21)$ regions, the predicted $S 6 / 10$ amino acid sequence was identical to several previously reported TIMP3 sequences $(19,20,22)$. Translation of the $56 / 10$ sequence indicated that it consists of $287 \mathrm{bp}$ of $5^{\prime}$-UTR sequence, $636 \mathrm{bp}$ of predicted coding sequence, and $3686 \mathrm{bp}$ of $3^{\prime}$ UTR sequence, representing 6,14 , and $80 \%$ of the $S 6 / 10$ sequence length, respectively (Fig. 2). The extensive $3^{\prime}$-UTR of the S6/10 sequence was compared with that of a $4.5-\mathrm{kb}$ murine TIMP3 
clone isolated by Sun et al. (16), and found to be $65 \%$ identical overall, with several regions of striking homology.

\section{Identification of the Use of Four Polyadenylation Sites Within the Human TIMP3 3'-UTR}

A comparison of the sequences of 3 smaller TIMP3 cDNAs isolated from the metastatic lymph node cDNA library with that of $56 / 10$ indicated the use of 4 different polyadenylation signals. Although the S1, S4, and S5 clones were all incomplete with respect to the $5^{\prime}$ end of S6/ 10 , their sequences were almost identical to S6/ 10 , except that each contained a polyA sequence commencing from a unique point along the S6/10 sequence. The corresponding polyadenylation signals (Fig. 2) were identified based on sequences and positions relative to the polyA sequence (27), and 3 of the 4 signals identified were found to be conserved in the murine TIMP3 $3^{\prime}$-UTR sequence (16). Interestingly, only the S4 clone indicated the use of a canonical AATAAA polyadenylation signal (Fig. 2).

\section{Expression Patterns of TIMP3 RNAs}

TIMP3 transcripts were detected by Northern blot analyses in 5 out of 7 normal tissues examined, most strongly in placenta and kidney, weakly in colon, lung and skin, but not in stomach or liver (data not shown). With in situ hybridization, TIMP3 transcripts were strongly detected within decidual cells of the endometrium in placental sections at 36 and 39 weeks gestation, and comparatively more weakly in some cells within the connective tissue of the placental villi (Fig. 3a and data not shown). Thus, the majority of TIMP3 transcripts detected within human placental RNA are likely to derive from decidual cells of the adjacent pregnant endometrium, rather than from placental tissue itself. In the kidney, TIMP3 transcripts were detected in endothelial cells of the small extraglomerular vasculature, and the glomeruli themselves were also weakly labeled (data not shown). TIMP3 transcripts were also localized to endothelial cells in corpus lutea, and in frontal and parietal cerebral cortex.

In breast carcinomas, TIMP3 transcripts were detected by Northern blot analysis in 8 out of 9 tumors, and, in 3 of these, transcript levels were clearly elevated compared with the expression in 7 out of 11 fibroadenomas (Fig. 1, and data not shown). With in situ hybridization, TIMP3 transcripts were predominantly detected in fibroblastic cells within the tumor stroma of $14 / 14$ breast carcinomas of different grades, representing 11 infiltrating ductal carcinomas, 3 infiltrating lobular carcinomas, and one medullary carcinoma (Fig. 3, and data not shown). Less intense fibroblastic labeling was also noted in 2 breast fibroadenomas (data not shown). In breast carcinomas, fibroblastic cells that were most strongly positive were frequently the ones immediately adjacent to islands of invasive cancer cells (Fig. 3 $\mathrm{c}$ and d) or surrounding carcinoma in situ found in the vicinity of infiltrating carcinoma (Fig. $3 \mathrm{e}$ through $\mathrm{h}$ ). When surrounded by intensely labeled fibroblastic cells, carcinoma cells were rarely labeled themselves. However, in some tissue sections cancer cells were weakly labeled, and in the vicinity of these, fibroblastic labeling was either reduced or not observed (data not shown). Similar weak levels of epithelial labeling were also observed in normal breast tissue adjacent to tumor areas.

FIG. 3. The distribution of TIMP3 transcripts in human breast carcinomas and normal tissues detected by in situ RNA hybridization

Bright field $(a-c, e, g)$ and dark field $(d, f, h)$ photomicrographs of paraffin-embedded tissue sections stained with toluidine blue after hybridization with ${ }^{35} \mathrm{~S}$-labeled antisense $(\mathrm{a}, \mathrm{c}-\mathrm{h})$ and sense (b) TIMP3 transcripts derived from a 1976-bp S6 TIMP3 subclone including 5'-UTR, coding sequences, and 1063 bp of 3'-UTR sequence are shown. Identical patterns of labeling were obtained using riboprobes derived from the entire 4579-bp TIMP3 cDNA (data not shown). (a) Placenta (36 weeks gestation) showing strong labeling of endometrial decidual cells. (b) The corresponding region of the same placental sample hybridized with the sense TIMP3 riboprobe. (c, d) An infiltrating ductal breast carcinoma showing strong labeling of fibroblastic cells in close contact with small islands of cancer cells, which are themselves not labeled. (e, f) In situ carcinoma of cribriform subtype, in which positive labeling is clearly associated with fibroblastic cells around the duct, while carcinoma cells within the duct are not labeled. (g, h) In situ carcinoma of micropapillary subtype, in which TIMP3 transcripts were strongly identified within stromal cells, while cells of the epithelial compartment were not labeled. Magnifications were $50 \times(\mathrm{g}, \mathrm{h}), 125 \times(\mathrm{a}, \mathrm{b}, \mathrm{e}, \mathrm{f})$, and $250 \times(\mathrm{c}, \mathrm{d})$. Exposure times were $14(\mathrm{a}, \mathrm{e}-\mathrm{h}), 21$ (c, d), and 32 days (b). 

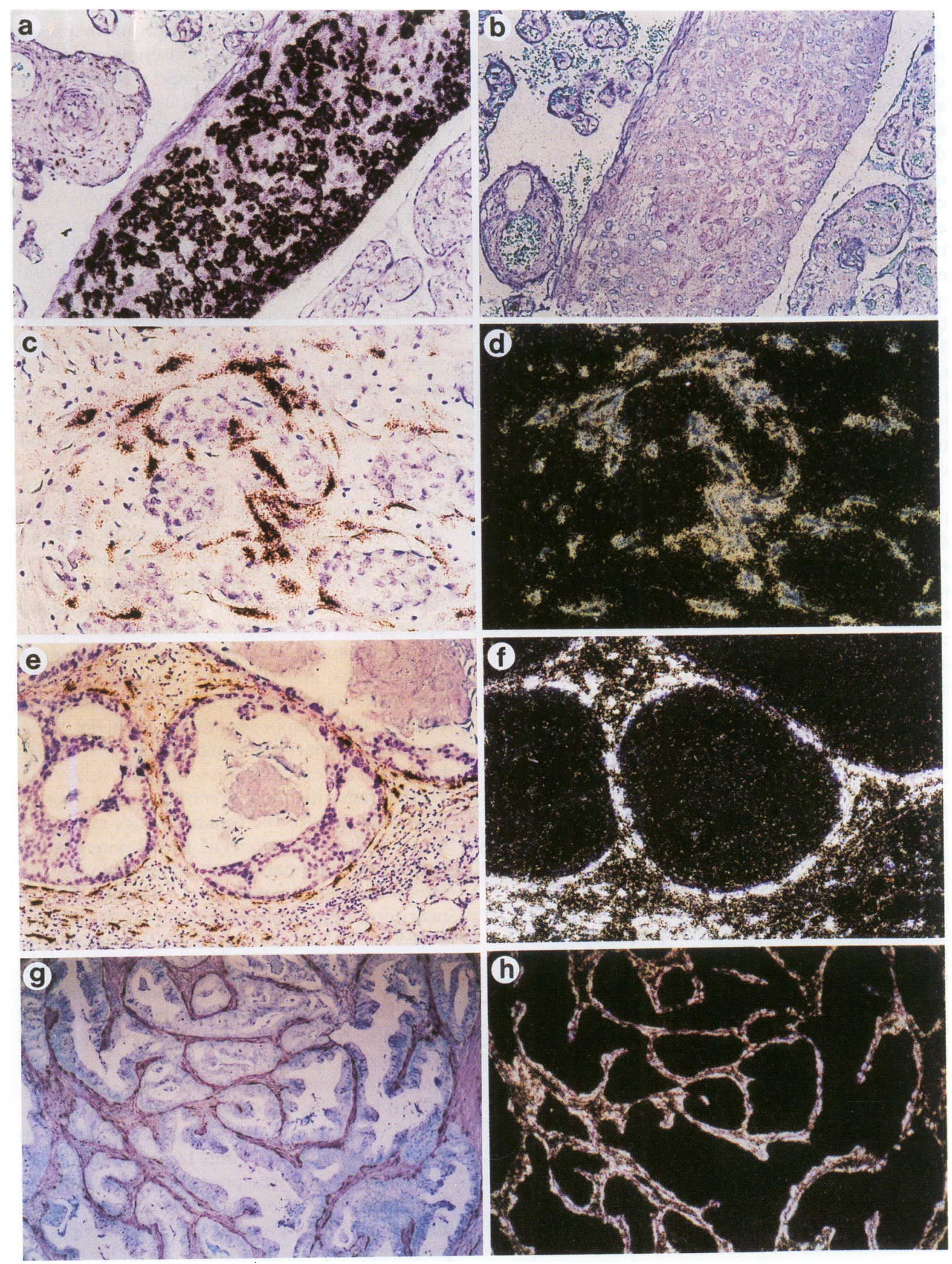


\section{DISCUSSION}

This study describes the cloning of cDNAs corresponding to 4 human TIMP3 transcripts, which include the TIMP3 transcripts $(4.6,2.7$, and 2.5 $\mathrm{kb})$ predominantly and most frequently detected in human tissues, as well as a less frequently detected $2.1-\mathrm{kb}$ TIMP3 transcript. The 4 sequences obtained in the present study differ from each other only with respect to their polyadenylation sites, indicating that these alternate TIMP3 transcripts arise from differential use of polyadenylation sites, rather than from alternative sequence use in other transcribed regions. The high proportions of both human and murine TIMP3 transcripts corresponding to 3'-UTR (16), and the fact that certain sequences within these regions are highly conserved, suggest that modulation of 3'-UTR length may represent a mechanism by which TIMP3 gene expression is controlled.

The use of the canonical AATAAA polyadenylation signal was indicated for only one of the 4 polyadenylation sites identified in the present study. Two hexanucleotide signals identified represented variants of the AATAAA sequence, namely ATTAAA and AGTAAA, which, in the context of SV40 late mRNAs, were found to lead to polyadenylation and cleavage with approximately 70 and $30 \%$ of the efficiency observed for the canonical polyadenylation signal, respectively (28). The remaining AGGAAA polyadenylation signal identified was classified as being nonrecognizable, differing by 2 nucleotides or more from the six nucleotide consensus (27), and the use of a similar AAGAAA signal was found to diminish the efficiency of polyadenylation by approximately $94 \%$ (28). Such hexanucleotide sequences are uncommonly encountered, and the AGGAAA polyadenylation signal was not found to be conserved in the murine TIMP3 3'-UTR (16).

The fact that variant signals are processed less efficiently than the canonical AATAAA sequence in vitro indicates that their use may provide a mechanism by which transcript abundance can be limited in vivo. The use of a variant polyadenylation signal AATATAA in an infrequently observed $1.2 \mathrm{~kb}$ human TIMP 3 transcript $(19,20,22)$ is in support of this concept. It is, however, believed that variant signals may operate more efficiently in vivo than predicted from in vitro studies, probably as a result of their contexts within the mRNA precursor sequence $(28,29)$. This would appear to be the case for the
AGGAAA and AGTAAA signals identified in the 2.7- and 4.6-kb TIMP3 transcripts in the present study. Both these TIMP 3 transcripts are readily detectable in a range of tissues by Northern blot analyses $(18,22)$, despite the use of polyadenylation signals which should significantly impair transcript cleavage and polyadenylation. Thus, it would appear that mRNA cleavage and polyadenylation alone do not limit the levels of all TIMP3 transcripts. The use of alternative polyadenylation signals to derive multiple TIMP3 transcripts may be significant in allowing the length and sequence composition of the extensive 3'-UTR to be modulated. Regardless, the choice between the various TIMP3 polyadenylation sites is likely controlled in part by cell- and/or tissue-specific factors, explaining the absence of particular TIMP3 transcripts in certain tissues and cell lines, as noted in this and previous studies $(17-19,21)$.

The present study represents the first instance in which in situ TIMP3 gene expression has been investigated in human tissues, although it has been examined in the developing mouse embryo at 12.5 and 14.5 days gestation, and in the newborn mouse (17). During mouse development, the expression of TIMP3 was noted to be prominent in the epithelial component of many organs, and TIMP3 was proposed to play a role in the development of skeletal and cardiac muscle, the morphogenesis of epithelial structures, and in placental implantation (17). In contrast, we observed in the present study that TIMP3 transcripts were predominantly expressed in stromal cells of the human tissues examined. In particular, the pattern of TIMP3 gene expression noted in human placenta was strikingly different from that noted in the mouse (17). In near-term human placental samples, TIMP3 was noted to be weakly expressed by some cells within the connective tissue of placental villi, but strongly expressed by the endometrial decidual cells. The decidual tissue is believed to control the degree of uterine invasion-for example, by synthesizing TIMPl (30). However, murine TIMP3 transcripts were observed by Apte et al. (17) to be solely expressed by the invasive placental component, the spongiotrophoblast, in placentas at 12.5, 14.5, and 16.5 days gestation.

While TIMP3 was previously demonstrated to be expressed in breast carcinomas by Uria et al. (20), we now show that TIMP3 transcripts are predominantly expressed by fibroblastic cells within the bed of these tumors. Labeling was frequently found to be most intense over fibro- 
blastic cells closest to the carcinoma cells, which themselves were infrequently found to express the TIMP3 gene. This contrasts with the pattern of expression noted for TIMP1 mRNA, which was detected in cancer cells of breast (31) and head and neck carcinomas (32). However, TIMP2 transcripts were localized to stromal cells in colorectal (33), head and neck (32), and breast carcinomas (34). Hence, the pattern of TIMP3 gene expression in breast carcinomas described in the present study appears to resemble that previously noted for TIMP2, where both genes appear to be predominantly stromally expressed.

In biological processes where net proteolytic enzyme activity is enhanced such as tumor development and angiogenesis, the concomitant synthesis of inhibitors is nonetheless required to regulate proteolytic activity (35-37). Expression of the TIMP 3 gene in the tumor stroma adjacent to cancer cells may therefore inhibit excessive local proteolysis of the stroma, particularly arising from the stromal cells themselves. In this respect, it is noteworthy that a number of extracellular proteinases implicated in carcinoma progression are expressed by fibroblastic cells with tissue distributions very similar to those of the fibroblastic cells expressing the TIMP3 gene $(38,39$ and references therein). In addition, TIMP 3 has been noted to promote the detachment of transforming chicken embryo fibroblasts from the extracellular matrix, as well as accelerating morphological changes associated with cell transformation (14). Thus, it could also be envisaged that TIMP3 facilitates the migration and accumulation of fibroblastic cells into the tumor stroma. This might, in turn, enhance cancer growth, since the tumor stroma is usually believed to be necessary for carcinoma expansion $(40,41)$. Thus, while a tumor suppressor function has been previously suggested for TIMP3 (16), it is equally possible that TIMP3 may function to promote tumor progression.

\section{ACKNOWLEDGMENTS}

We are indebted to P. Chambon for his support and interest in this study, and we thank J.-M. Garnier and S. Ward for discussions, and I. Stoll and S. Vicaire for excellent technical assistance.

This work was supported by funds from the Institut de la Santé et de la Recherche Médicale, the Centre National de la Recherche Scientifique, the Centre Hospitalier Universitaire Régional, the Mutuelle Générale de l'Education Nationale, the Ministère de la Recherche et de l'Espace
(Grants 92H/0917 and 92N60/0694 to J.-L. Mandel), the Association pour la Recherche sur le Cancer, the Ligue Nationale Française contre le Cancer, the Fondation pour la Recherche Médicale Française, and a grant to $\mathrm{P}$. Chambon from the Fondation Jeantet. JAB is the recipient of a C. J. Martin Postdoctoral Fellowship from the National Health and Medical Research Council of Australia.

\section{REFERENCES}

1. Pavloff N, Staskus PW, Kishnani NS, Hawkes SP. (1992) A new inhibitor of metalloproteinases from chicken: chIMP-3. J. Biol. Chem. 267: 17321-17326.

2. Boone T, Johnson MJ, De Clerck YA, Langley KE. (1990) cDNA cloning and expression of a metalloproteinase inhibitor related to tissue inhibitor of metalloproteinases. Proc. Natl. Acad. Sci. U.S.A. 87: 2800-2804.

3. Matrisian LM. (1992) The matrix-degrading metalloproteinases. BioEssays 14: 455-463.

4. Alexander CM, Werb Z. (1991) Extracellular matrix degradation. In: Hay ED (ed). Cell Biology of Extracellular Matrix. Plenum Press, New York, pp. 255-302.

5. Birkedal-Hansen H, Moore WGI, Bodden MK, et al. (1993) Matrix metalloproteinases: A review. Critic. Rev. Oral Biol. Med. 4: 197250.

6. Denhardt DT, Feng B, Edwards DR, Cocuzzi ET, Malyankar UM. (1993) Tissue inhibitor of metalloproteinases (TIMP, aka EPA): Structure, control of expression and biological functions. Pharmacol. Ther. 59: 329-341.

7. Jones SE, Jomary C, Neal MJ. (1994) Expression of TIMP 3 mRNA is elevated in retinas affected by simplex retinitis pigmentosa. F.E.B.S. Lett. 352: 171-174.

8. Weber BHF, Vogt G, Pruett RC, Stöhr H, Felbor U. (1994) Mutations in the tissue inhibitor of metalloproteinases-3 (TIMP3) in patients with Sorsby's fundus dystrophy. $\mathrm{Na}$ ture Genet. 8: 352-356.

9. Docherty AJP, O'Connell J, Crabbe T, Angal S, Murphy G. (1992) The matrix metalloproteinases and their natural inhibitors: Prospects for treating degenerative tissue diseases. Trends Biotechnol. 10: 200-207.

10. Gasson JC, Golde DW, Kaufman SE, et al. (1985) Molecular characterization and expression of the gene encoding human eryth- 
roid-potentiating activity. Nature 315: 768771.

11. Hayakawa T, Yamashita K, Tanzawa K, Uchijima E, Iwata K. (1992) Growth-promoting activity of tissue inhibitor of metalloproteinases-1 (TIMP-1) for a wide range of cells. A possible new growth factor in serum. F.E.B.S. Lett. 298: 29-32.

12. Stetler-Stevenson WG, Bersch N, Golde DW. (1992) Tissue inhibitor of metalloproteinase-2 (TIMP-2) has erythroid potentiating activity. F.E.B.S. Lett. 296: 231-234.

13. Murphy AN, Unsworth EJ, Stetler-Stevenson WG. (1993) Tissue inhibitor of metalloproteinases- 2 inhibits bFGF-induced human microvascular endothelial cell proliferation. J. Cell. Physiol. 157: 351-358.

14. Yang T-T, Hawkes SP. (1992) Role of the 21-kDa protein TIMP-3 in oncogenic transformation of cultured chicken embryo fibroblasts. Proc. Natl. Acad. Sci. U.S.A. 89: 1067610680.

15. Leco KJ, Khokka R, Pavloff N, Hawkes SP, Edwards DR. (1994) Tissue inhibitor of metalloproteinases-3 (TIMP-3) is an extracellular matrix-associated protein with a distinctive pattern of expression in mouse cells and tissues. J. Biol. Chem. 269: 9352-9360.

16. Sun Y, Hegamyer G, Colburn N. (1994) Molecular cloning of five messenger RNAs differentially expressed in preneoplastic or neoplastic JB6 mouse epidermal cells: One is homologous to human tissue inhibitor of metalloproteinases-3. Cancer Res. 54: 11391144.

17. Apte SS, Hayashi K, Seldin MF, Mattei M-G, Hayashi M, Olsen BR. (1994) Gene encoding a novel murine tissue inhibitor of metalloproteinases (TIMP), TIMP-3, is expressed in developing mouse epithelia, cartilage, and muscle, and is located on mouse chromosome 10. Dev. Dynam. 200: 177-197.

18. Apte SS, Mattei M-G, Olsen BR. (1994) Cloning of the cDNA encoding human tissue inhibitor of metalloproteinases-3 (TIMP-3) and mapping of the TIMP3 gene to chromosome 22. Genomics 19: 86-90.

19. Silbiger SM, Jacobsen VL, Cupples RL, Koski RA. (1994) Cloning of cDNAs encoding human TIMP-3, a novel member of the tissue inhibitor of metalloproteinase family. Gene 141: 293-297.

20. Uria JA, Ferrando AA, Velasco G, Freije JMP, Lopez-Otin C. (1994) Structure and expression in breast tumors of human TIMP-3, a new member of the metalloproteinase inhibitor family. Cancer Res. 54: 2091-2094.

21. Wick M, Burger C, Brusselbach S, Lucibello F, Muller R. (1994) A novel member of human tissue inhibitor of metalloproteinases (TIMP) gene family is regulated during $G^{1}$ progression, mitogenic stimulation, differentiation and senescence. J. Biol. Chem. 269: 18953-18960.

22. Wilde CG, Hawkins PR, Coleman RT, et al. (1994) Cloning and characterization of human tissue inhibitor of metalloproteinases-3. DNA Cell Biol. 13: 711-718.

23. Byrne JA, Tomasetto C, Garnier JM, et al. A screening method to identify genes commonly overexpressed in carcinomas, and the identification of a novel cDNA sequence. Cancer Res. (In Press.).

24. Davis MM, Cohen DI, Nielsen EA, Steinmetz M, Paul WE, Hood L. (1984) Cell-type-specific cDNA probes and the murine I region: The localization and orientation of $\mathrm{A}_{\alpha}{ }^{\mathrm{d}}$. Proc. Natl. Acad. Sci. U.S.A. 81: 2194-2198.

25. Basset P, Bellocq JP, Wolf C, et al. (1990) A novel metalloproteinase gene specifically expressed in stromal cells of breast carcinomas. Nature 348: 699-704.

26. Masiakowski P, Breathnach R, Bloch J, Gannon F, Krust A, Chambon P. (1982) Cloning of a cDNA sequence of hormone-regulated genes from the MCF-7 human breast cancer cell line. Nucleic Acids Res. 10: 7895-7903.

27. Wahle E, Keller W. (1992) The biochemistry of $3^{\prime}$-end cleavage and polyadenylation of messenger RNA precursors. Annu. Rev. Biochem. 61: 419-440.

28. Sheets MD, Ogg SC, Wickens MP. (1990) Point mutations in AAUAAA and the poly (A) addition site: Effects on the accuracy and efficiency of cleavage and polyadenylation in vitro. Nucleic Acids Res. 18: 5799-5805.

29. Wilusz J, Pettine SM, Shenk T. (1989) Functional analysis of point mutations in the AAUAAA motif of the SV40 late polyadenylation signal. Nucleic Acids Res. 17: 3989-3908.

30. Graham CH, Lala PK. (1992) Mechanisms of placental invasion of the uterus and their control. Biochem. Cell Biol. 70: 867-874.

31. Polette $M$, Clavel C, Cockett $M$, Girod de Bentzmann S, Murphy G, Birembaut P. (1993) Detection and localization of mRNAs encoding matrix metalloproteinases and their tissue inhibitor in human breast pathology. Invasion Metastasis 13: 31-37.

32. Polette M, Clavel C, Birembaut P. (1993) 
Localization by in situ hybridization of mRNAs encoding stromelysin-3 and tissue inhibitors of metalloproteinases TIMP- 1 and TIMP-2 in human head and neck carcinomas. Path. Res. Pract. 189: 1052-1057.

33. Poulsom R, Pignatelli $M$, Stetler-Stevenson WG, et al. (1992) Stromal expression of 72 kda type IV collagenase (MMP-2) and TIMP-2 mRNAs in colorectal neoplasia. Am. J. Pathol. 141: 389-396.

34. Poulsom R, Hanby AM, Pignatelli $M$, et al. (1993) Expression of gelatinase $A$ and TIMP-2 mRNAs in desmoplastic fibroblasts in both mammary carcinomas and basal cell carcinomas of the skin. J. Clin. Pathol. 46: 429-436.

35. Woessner JF. (1991) Matrix metalloproteinases and their inhibitors in connective tissue remodelling. F.A.S.E.B. J. 5: 2145-2146.

36. Mignatti P, Rifkin D. (1993) Biology and

Contributed by P. Chambon on March 3, 1995. biochemistry of proteinases in tumor invasion. Physiol. Rev. 73: 161-195.

37. Stetler-Stevenson WG, Aznavoorian S, Liotta LA. (1993) Tumor cell interactions with the extracellular matrix during invasion and metastasis. Annu. Rev. Cell Biol. 9: 541-573.

38. Wolf C, Rouyer N, Lutz Y, et al. (1993) Stromelysin 3 belongs to a subgroup of proteinases expressed in breast carcinoma cells and possibly implicated in tumor progression. Proc. Natl. Acad. Sci. U.S.A. 90: 1843-1847.

39. Okada A, Bellocq JP, Rouyer N, et al. (1995) Membrane-type metalloproteinase (MT-MMP) gene is expressed in stromal cells of human colon, breast and head and neck carcinomas. Proc. Natl. Acad. Sci. U.S.A. 92:2730-2734.

40. Dvorak HF. (1987) Tumors: Wounds that do not heal. N. Engl. J. Med. 315: 1650-1659.

41. Zipori D. (1990) Stromal cells in tumor growth and regression. Cancer J. 3: 164-169. 